\section{Targeting out-of-hospital cardiac arrest: the effect of heparin administered during cardiopulmonary resuscitation (T-ARREST)}

JIŘí KNOR • MILANA POKORNÁ • ROMAN ŠKULEC • JIŘí MÁLEK • PETR WALDAUF・ROMAN SKŘIPSKÝ • VLADIMÍR ČERNÝ
JIŘí KNOR ( $\square)$

The Regional Centre of the Emergency Medical Service of the Central Bohemian Region Emergency Medical Service of the Capital City of Prague Department of Anaesthesiology and Resuscitation of the $3^{\text {rd }}$ Medical Faculty Charles University in Prague and Faculty Hospital Královské Vinohrady USZSSK, Vancurova 1544, Kladno Hospital, Kladno 266 01, Czech Republic Phone: 00420731137004 E-mail: jiri.knor@centrum.cz

MILANA POKORNÁ • ROMAN SKŘIPSKÝ Emergency Medical Service of the Capital City of Prague, Czech Republic

ROMAN ŠKULEC

The Regional Centre of the Emergency Medical Service of the Central Bohemian Region, Czech Republic and Department of Anaesthesiology and Intensive Care, Charles University in Prague, Faculty of Medicine in Hradec Kralove, University Hospital Hradec Kralove, Czech Republic

JIŘí MÁLEK • PETR WALDAUF Department of Anaesthesiology and Resuscitation of the $3^{\text {rd }}$ Medical Faculty, Charles University in Prague and Faculty Hospital Královské Vinohrady, Czech Republic

VLADIMÍR ČERNÝ

Department of Anesthesiology and Intensive Care, Charles University in Prague, Faculty

of Medicine in Hradec Kralove, University Hospital Hradec Kralove, Czech Republic and Department of Anesthesia, Dalhousie

University, Halifax, Canada

\begin{abstract}
Introduction. Heparin administration during cardiopulmonary resuscitation (CPR) may prevent activation of coagulation after successful resuscitation for out-of-hospital cardiac arrest (OHCA). We hypothesize that such an approach is not associated with an increased rate of bleeding, but it has not been evaluated. We performed a pilot randomized clinical study assessing the safety of intra-arrest heparin administration in OHCA patients with suspected acute myocardial infarction (AMI) and its impact on their prognosis.

Materials and Methods. OHCA patients were randomized during CPR to 10000 units of intra-arrest intravenous heparin (Group $H$ ) or to treatment without heparin (Group C). The occurrence of major bleeding and the presence of a favourable neurological result 3 months after OHCA, were analyzed.

Results. Out of 88 randomized patients, AMI was subsequently confirmed in 63 of them (71.6\%). There were 30 patients in group $\mathrm{H}$ and 33 in group $\mathrm{C}$. No major bleeding event was observed in either group. Return of spontaneous circulation (ROSC, Group H: 40.0\%, Group C: 45.4\%, $p=0.662$ ) and a good neurological result 3 months after OHCA (Group H: $6.7 \%$, Group C: $9.1 \%, p=0.921$ ) did not differ between groups.

Conclusions. Intravenous administration of 10000 units of heparin during CPR for OHCA in patients with supposed AMI was safe. We did not find any improvement in prognosis for our sample of limited size. Though the procedure proved safe, we recommend postponing the administration of heparin until ROSC, assessment of clinical state and recording of a twelve-lead ECG.
\end{abstract}


Key words: out-of-hospital cardiac arrest, heparin, major bleeding

\section{Introduction}

Out-of-hospital cardiac arrest (OHCA) is a critical condition with an uncertain prognosis. Even if effective cardiopulmonary resuscitation (CPR) is started, a number of complex pathophysiological mechanisms are activated during the arrest and after it, resulting in the development of ischemia-reperfusion injury and post-cardiac arrest syndrome. (1) One of these processes is systemic coagulation activation with subsequent fibrin formation. In conjunction with other processes, such as activation of leucocytes, endothelial dysfunction and increased blood viscosity, this can lead to microcirculatory dysfunction and no-reflow phenomenon. (2-4) One of the theoretical possibilities of how to stop the spiral of ischemia-reperfusion injuries is the preventive administration of a thrombolytic and/or anticoagulant agent during CPR. Moreover, in the case of a cardiac aetiology of OHCA, the primary thrombotic episode in the coronary or pulmonary artery may also be a potential target of this therapy. While systemic thrombolysis during OHCA has been studied, to the best of our knowledge, there has been no published clinical trial evaluating the preventive impact of sole heparin administration during CPR. $(3,5)$ We hypothesize that such an approach is safe and not associated with an increased rate of bleeding. However, this treatment has not been included in the CPR guidelines. $(6,7)$ Thus, it should be assessed by a clinical study.

Therefore, we decided to perform a pilot randomized clinical study to assess the safety of administering non-fractionated heparin during CPR for OHCA to patients presumed to have acute myocardial infarction (AMI) and also to analyze its impact on their prognosis.

\section{Materials and Methods}

The clinical study was carried out in accordance with the Declaration of Helsinki and its realization was approved by the ethics committee. Informed consent was waived for the clinical condition of the patients with cardiac arrest. The study was the first part of the project T-ARREST working group (Targeting Out-of-Hospital Cardiac Arrest). Patients treated by the City of Prague Emergency Medical Service were included. The protocol of the study was carried out during emergency pre-hospital care and contained two decisionmaking moments (figure 1). In the first stage, the attending physician of the Emergency Medical Service decided, usually immediately upon arrival, if the patient having an OHCA is a suitable prospective candidate for randomization. The criteria for including or not including the patients for randomisation are summarized in table 1. The diagnosis of acute myocardial infarction, as a supposed cause of OHCA, was done on the basis of the following conditions: 1. the absence of signs of bleeding and trauma and 2. the collapse was preceded by the sudden onset of chest pain (or another type of sudden pain as left or right arm, abdominal or neck pain) and/or with dyspnoea or 3. the patient exhibited any prodromal symptoms before collapse and/or 4. known history of coronary artery disease.

CPR was always performed in accordance with European resuscitation council guidelines. After completing all routine procedures specified by standard protocols (continuous ECG monitoring, endotracheal intubation, venous access, initial defibrillation if indicated, continuous monitoring of peripheral oxygen saturation $\mathrm{SpO}_{2}$ and end tidal $\mathrm{CO}_{2}\left(\mathrm{EtCO}_{2}\right)$, the patients were randomized (by random numbers generator) during ongoing CPR to a intravenous bolus administration of non-fractionated heparin (Heparin, Leciva, inj. sol.) in a dose of 10000 units (Group H) or to continuing standard resuscitation without the administration of heparin during the procedure (Group C). For both groups, CPR was performed for at least 30 minutes or until return of spontaneous circulation (ROSC). In the case of ROSC, patients were transported to hospitals where the method of direct percutaneous coronary intervention was available. Subsequent in-hospital therapy was standard and not influenced by the study protocol. Clinical course was followed up according to the Utstein protocol. Finally, the diagnosis of acute myocardial infarction was reassessed for all patients (clinically during hospitalization or by post-mortem examination).

The patients whose spontaneous circulation returned before completing all monitoring and therapeutic procedures and the patients who failed to undergo all the above mentioned procedures were not randomized. These patients were not followed up.

Artificial ventilation during and after CPR was carried out in a routine way with the respiration rate aproximately $14 / \mathrm{min}$, the tidal volumes $7-10 \mathrm{ml} . \mathrm{kg}^{-1}$ and oxygen inspiratory fraction 1.0 (MEDUMAT Standard, Weinmann medical technology, Hamburg, Deutschland). Monitoring of $\mathrm{EtCO}_{2}$ was performed using the mainstream technique (Zoll's M Series defibrillator, Zoll Medical Corporation, Chelmsford, MA, USA). Monitoring of ECG and electric impulse therapy was also ensured by the Zoll apparatus and all measurements were saved on the memory card of the apparatus providing precise feedback and assessment of the resuscitation. Midazolam combined with fentanyl or sufentanyl were used for analgesia and sedation of patients. Pipecuronium was used for muscle relaxation, if needed. All patients who achieved ROSC were subsequently administered lysine salicylate intravenously. Patients in Group $\mathrm{C}$ were not given heparin in the prehospital stage of treatment even after achieving ROSC.

\section{Endpoints}

The primary endpoint was the presence of major bleeding in the pre-hospital stage of treatment or during the first 48 hours of hospitalization, as a safety measure. Major bleeding was defined as a decrease in haemoglobin levels of $>20 \mathrm{~g} \mathrm{I}^{-1}$, need for transfusion of $>2$ units of blood, need for corrective surgery, intracranial or retroperitoneal haemorrhage, or any combination of 
Table 1. Inclusion and exclusion criteria for randomization.

\begin{tabular}{l}
\hline INCLUSION CRITERIA FOR RANDOMIZATION* \\
\hline - out-Of-hospital cardiac arrest \\
\hline - supposed aetiology of acute myocardial infarction \\
\hline - starting cardiopulmonary resuscitation \\
\hline EXCLUSION CRITERIA FOR RANDOMIZATION** \\
\hline
\end{tabular}

- possible or certain traumatic, bleeding or other non-infarction aetiology of cardiac arrest

- absence of a physician at the emergency medical service at the start of cardiopulmonary resuscitation

- pre-defined status not to intubate and/or resuscitate

Note: *...must meet all criteria, ${ }^{* \star}$...must not meet any of the criteria

Table 2. Clinical causes of OHCA, ROSC rate, three-month mortality and the neurological result in patients with other aetiology of OHCA, other than AMI. OHCA, out-of-hospital cardiac arrest; AMI, acute myocardial infarction; ROSC, return of spontaneous circulation; CPC, cerebral performance category.

\begin{tabular}{clll}
\hline & GROUP H & GROUP C & $\mathrm{p}$ \\
\hline Number of patients (n) & 11 & 14 & \\
\hline Clinical cause of the arrest & & & \\
\hline Pulmonary oedema (n (\%)) & $4(36.4)$ & $4(28.6)$ & 0.987 \\
\hline Pulmonary embolism (n (\%)) & $4(36.4)$ & $3(21 ., 4)$ & 0.704 \\
\hline Pulmonary disease (n (\%)) & $3(27.2)$ & $5(35.7)$ & 0.984 \\
\hline Other (n (\%)) & 0 & $2(14.3)$ & 0.572 \\
\hline Achieved ROSC (n (\%)) & $6(54.5)$ & $6(42.3)$ & 0.839 \\
\hline Three-month mortality (n (\%)) & $6(54.5)$ & $10(71.4)$ & 0.650 \\
\hline CPC 1 or 2 after 3 months (n (\%)) & $2(18.2)$ & $1(7.1)$ & 0.820 \\
\hline CPC 3 or 4 after 3 months (n (\%)) & $3(27.3)$ & $3(21.4)$ & 0.897 \\
\hline
\end{tabular}

OHCA, out-of-hospital cardiac arrest; AMI, acute myocardial infarction; ROSC, return of spontaneous circulation; CPC,cerebral performance category.

these events diagnosed clinically or by post-mortem examination. (8) The secondary endpoints were mortality rate and recovering with a favourable neurological result 3 months after cardiac arrest. The neurological result was assessed according to the Cerebral Performance Category (CPC) marking system. (9) Categories 1 and 2 were considered a favourable result.

\section{Statistical analysis}

The values of monitored parameters were expressed as average values \pm standard deviation (SD) or as median (minimal - maximal value) or by percentage. The differences between unpaired groups in case of alternative variables were compared by Pearson's chi-square test and in case of continual variables by Student's t-test. The differences between groups with non-normal data distribution were compared by Wilcoxon rank sum test. The statistics were processed using Microsoft Excel 2007 programme (Microsoft, Redmond, WA, USA) and JMP 3.2 statistical software (SAS Institute, Cary, NC, USA). P value $<0.05$ was considered statistically significant.

\section{Results}

From January 2004 till December 2006 there were 141 prospective candidates chosen for randomization. 88 of them (62.4\%) were randomized. The remaining 53 patients either achieved ROSC before meeting technical conditions for randomization or it was not possible to ensure reliably all monitoring procedures required by the protocol and preceding the randomization (most often reliable monitoring of $\mathrm{EtCO}_{2}$ or oxygen saturation).

AMI was subsequently confirmed in 63 out of 88 randomized patients ( 71.6 $\%)$. In the group of randomized patients who had another cause of OHCA other than AMI (as confirmed afterwards), 11 were given heparin during CPR and 14 patients were not. Major bleeding (gastrointestinal) was observed at postmortem examination in one patient who had been given heparin. Clinical causes of arrest, the primary success rate of CPR and the neurological result and mortality during the three months after the arrest are presented in table 2 .

In the group of randomized patients with confirmed AMI, 30 were randomized to Group H and 33 patients to Group C. These patients were subject to detailed analysis. Basic demographic data, the 
Table 3. Demographic data, initial rhythm and characteristics of CPR.

\begin{tabular}{|c|c|c|c|}
\hline & GROUP H & GROUP C & $\mathrm{p}$ \\
\hline $\begin{array}{l}\text { Number of patients in the } \\
\text { group }(n)\end{array}$ & 30 & 33 & \\
\hline Men in the group ( $\mathrm{n}(\%))$ & $25(83.3)$ & $30(90.1)$ & 0.367 \\
\hline Age (median (min-max)) & $65.0(27-85)$ & $64.0(20-82)$ & $0.934^{*}$ \\
\hline $\begin{array}{l}\text { Estimated body weight } \\
(\mathrm{kg} \pm \mathrm{SD})\end{array}$ & $84 \pm 13$ & $89 \pm 16$ & 0.181 \\
\hline $\begin{array}{l}\text { Any bystander CPR attempt (n } \\
(\%))\end{array}$ & $12(40.0)$ & $13(39.4)$ & 0.961 \\
\hline $\begin{array}{l}\text { Any symptoms preceding } \\
\text { OHCA (median (min-max) }\end{array}$ & $12(40.0)$ & $14(42.4)$ & 0.845 \\
\hline VF as the initial rhythm (n (\%)) & $27(90.0)$ & $30(90.1)$ & 0.902 \\
\hline $\begin{array}{l}\text { Time from collapse to any } \\
\text { resuscitation attempt (min } \pm S D)\end{array}$ & $4.3 \pm 3.1$ & $4.5 \pm 3.4$ & 0.807 \\
\hline $\begin{array}{l}\text { Time from collapse to ROSC/ } \\
\text { end of CPR ( } \min \pm S D)\end{array}$ & $38 \pm 22$ & $29 \pm 12$ & 0.046 \\
\hline $\begin{array}{l}\text { Cumulative defibrillation ener- } \\
\text { gy in } \\
\text { ventricular fibrillation patients } \\
(\mathrm{J}, \\
\text { median (min-max)) }\end{array}$ & $\begin{array}{l}560 \\
(0-2520)\end{array}$ & $\begin{array}{l}560 \\
(0-2880)\end{array}$ & $0.590^{*}$ \\
\hline $\begin{array}{l}\text { Cumulative epinephrine dose } \\
\text { (mg, } \\
\text { median (min.max)) }\end{array}$ & $4.0(0-14)$ & $4.0(0-13)$ & $0.787^{*}$ \\
\hline Achieved ROSC (n (\%)) & $12(40.0)$ & $15(45.4)$ & 0.662 \\
\hline $\begin{array}{l}\text { Recurrence of cardiac arrest } \\
\text { during } \\
\text { transport }(\mathrm{n}(\%))\end{array}$ & $2(6.7)$ & $3(6.1)$ & 0.722 \\
\hline $\begin{array}{l}\text { Ongoing CPR on arrival at } \\
\text { hospital } \\
(\mathrm{n}(\%))\end{array}$ & $1(7.5)$ & $1(12.5)$ & 0.945 \\
\hline
\end{tabular}

CPR, cardiopulmonary resuscitation; max, maximal value; min, minimal value; ROSC, return of spontaneous circulation; OHCA, out-of-hospital cardiac arrest; SD, standard deviation; $\mathrm{VF}$, ventricular fibrillation

Table 4. Three-month mortality and patients' neurological results. CPC, cerebral performance category.

\begin{tabular}{llll}
\hline & GROUP H & GROUP C & $p$ \\
\hline Three-month mortality (n (\%)) & $26(86.6)$ & $26(78.8)$ & 0.411 \\
\hline $\begin{array}{l}\text { Achieved CPC 1 or 2 after 3 mon- } \\
\text { ths (n (\%)) }\end{array}$ & $2(6.7)$ & $3(9.1)$ & 0.921 \\
\hline $\begin{array}{l}\text { Achieved CPC 3 or 4 after 3 mon- } \\
\text { ths (n (\%)) }\end{array}$ & $2(6.7)$ & $4(12.1)$ & 0.885 \\
\hline
\end{tabular}

initial rhythm and parameters describing cardiopulmonary resuscitation are presented in table 3. Ventricular fibrillation, as an initial rhythm, was found in the majority of patients in both groups, all remaining patients had asystole. Only a minority of patients presented prodromal pre-arrest symptoms, namely chest pain (group H: $20.0 \%$, group $\mathrm{C}: 21.2 \%, \mathrm{p}=0.905)$, nausea (group $\mathrm{H}$ : $13.3 \%$, group C: $12.1 \%, p=0.885)$ and dyspnoea (group $\mathrm{H}: 6.7 \%$, group C: $9.1 \%, p=0.722$ ). AMl was diagnosed in most of them as a probable cause (in Group H: 60.0 \%, in Group C: 57.6 $\%, p=0.735)$.

Major bleeding was not observed in any patient in Group H and in Group C.

There were no statistical differences between groups in regard to having a favourable neurological result and mortality 3 months after the OHCA (table 4). In the subgroup of patients who achieved ROSC in the pre-hospital stage of treatment, a favourable neurological result 3 months after the OHCA was reached with comparable frequency as well (Group H: 16.7 \%, Group C: $20.0 \%, p=0.825)$.

\section{Discussion}

The main finding of our study is that the administration of heparin during cardiopulmonary resuscitation for $\mathrm{OHCA}$ as a consequence of acute myocardial infarction is a safe procedure, not associated with an increased number of major bleeding events. Its impact on long-term prognosis is not clear. Out-of-hospital cardiac arrest is an extremely serious medical event. Only approximately $11 \%$ of patients survive to be discharged from hospital. $(10,11)$ One of the procedures that may possibly improve the clinical results of resuscitated OHCA patients is very early pharmacological intervention during $\mathrm{CPR}$, potentially having an influence on the cause of OHCA and also on subsequent post-resuscitation processes. Some mechanisms of ischemia-reperfusion injury are activated as early as during CPR. Significant activation of thrombocytes and coagulation cascades without corresponding increase 
of fibrinolytic activity are also among them. (12) This considerable imbalance favouring blood clotting may lead to microcirculation dysfunction based both on the vasoactive basis and microthrombi formation in tissues. This was described especially in the brain and the impairment of coagulation activity in the microvascular compartment has been considered to be one of the main conditions leading to the cerebral no-reflow phenomenon. (3) The degree of no-reflow probably translates into the neurological outcome. A decreased possibility of spontaneous reperfusion of the thrombotic closure of the coronary artery may also play a role. Observational studies assessing the importance of echocardiography during prolonged resuscitation showed that the presence of a spontaneous echo contrast during CPR was connected with a worse prognosis. (13-15) All of these lead to the reduction of oxygen supplies in tissues and contribute to the development of the post-cardiac arrest syndrome and subsequent organ dysfunctions. $(2,16)$ In theory, this unfavourable state may be positively modified by antithrombotic treatment.

The isolated effect of the antiaggregant treatment on the processes during and after cardiac arrest has been studied only to a very limited extent. (17-22) In general, these are experimental receptor studies. No trials evaluating the safety and/or clinical effectiveness of antiaggregants administered during cardiac arrest have been published. With regards to the boom of new and very effective antiaggregants, with the possibility of intravenous administration, there is much space left for research into their effect in patients with cardiac arrest.(23)

The attention of researches has been recently focused on the study of the effect of systemic thrombolysis given during CPR on patients' prognosis. (24-30) Bottiger et al. have carried out an extensive multicentric clinical trial TROICA (Thrombolysis Using Tenecteplase in Cardiac Arrest). They randomized 1050 patients with OHCA of supposed cardiac aetiology to the adminis-

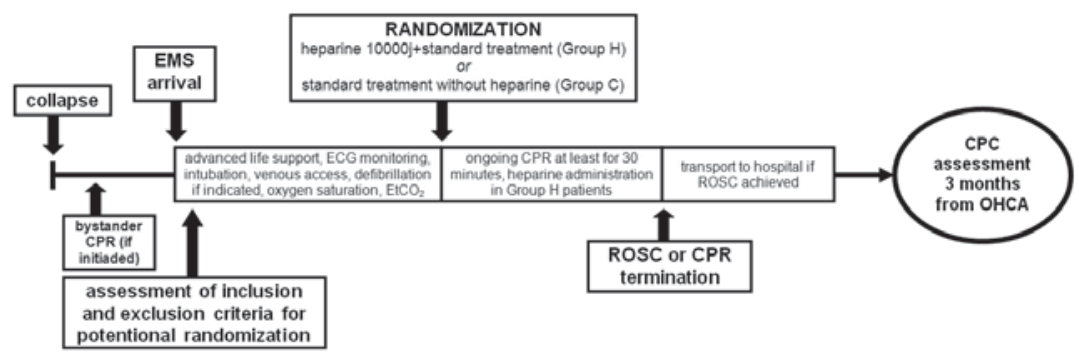

Figure 1. Study protocol schedule.

CPR, cardiopulmonary resuscitation; EMS, emergency medical service; OHCA, out-of-hospital cardiac arrest; ROSC, return of spontaneous circulation

tration of tenecteplase bolus or placebo during CPR. The authors did not find any difference in thirty-day survival (14.7 versus $17.0 \%, p=0,36)$. (27)

The only bleeding event found in the study was an incidence of intracranial bleeding. It was increased in the tenecteplase group (2.7 versus $0.4 \%$, $p=0.006$ ). However, in some patients the bleeding was a cause of cardiac arrest and most of the patients were asymptomatic. Since indications for cranial computer scanning were not predefined, detection bias cannot be ruled out. A metaanalysis of small studies and case reports revealed data about the safety of the administration of a thrombolytic agent during CPR. (31) We decided to evaluate the safety and effect of heparin administered during CPR for several reasons. Firstly, the influence of heparin on the success rate of CPR has been studied insufficiently in a similar way as the effect of antiaggregant treatment. Only certain positive results were observed in animal experiments. $(32,33)$ In clinical practice, there are only studies combining heparin with system thrombolysis and a case reportconcerning the administration of heparin. $(27,34)$ Secondly, it is important that ambiguous results of thrombolytic studies do not automatically disqualify a possible profit from the administration of heparin. The effect of heparin on the coagulation and fibrinolytic system is different from the effect of thrombolysis and from the point of view of pathophysiology it may be used especially as intracardiac thrombosis prevention during arrest and microcirculation dysfunction. (34) Thirdly, the administration of heparin is very simple and it is an inexpensive drug. Being a pilot study, we mainly focused on the safety of such an approach, expressed by the incidence of major bleeding events.

The first important point is the diagnosis of a possible cardiac cause of OHCA itself. It is necessary to disqualify any traumatic cause and/or bleeding. The information about the medical history of the patients and about any prodromal symptoms that directly preceded $\mathrm{OHCA}$ (if there were any at all) is not always available. Usually, it is necessary to make a diagnosis based on the result of a rapid examination of an unconscious patient with cardiac arrest and to use the process of elimination. Acute myocardial infarction is the cause of OHCA in one half to two thirds of all cases of OHCA after eliminating apparently non-cardiac causes, that is traumatic causes and/or bleeding etc. $(35,36)$ The number of patients with AMI would be probably even higher after eliminating patients supposed to have pulmonary embolism. In our group, respecting the inclusion criteria, the diagnosis of acute myocardial infarction was confirmed later in $71.6 \%$ of cases. This relatively high confidence level of the initial diagnosis confirms the above mentioned observations and at the same time makes it possible in a relatively reliable way to identify the most suitable target group of patients with OHCA for anti-thrombotic intervention during $\mathrm{CPR}$. 
The key finding of our study is that the use of heparin during CPR was not associated with an increase in major bleeding events during the pre-hospital and early in-hospital period. Even in those patients who did not reach ROSC in the field, no bleeding events, potentially worsening the chance to achieve ROSC, were found. Therefore, we consider the administration of unfractionated heparin during CPR in OHCA patients with presumed AMI safe.

We did not observe any impact of heparin administration on the threemonth mortality and on the incidence of a good neurological outcome in our study. There are several possible reasons for this finding: Is it a small number of patients in the study? Is it the absence of any effect of heparin, an unsuitable study design, an incorrect dosage of heparin or a choice of unsuitable endpoints? All of the above mentioned elements may influence the result. We are sure that more patients included would be beneficial, however, the outcome was not the primary endpoint. An optimal way would be to perform a double-blinded clinical study with a high number of randomized patients and thoroughly defined prehospital and hospital care, with heparin dosages in units $/ \mathrm{kg}$ of body weight and a technical primary endpoint directly assessing the coagulation activity and the state of microcirculation. Nevertheless, such a study would be extremely demanding in regard to financing, time and organization, especially in view of the fact that the study concerns a pre-hospital intervention in patients with OHCA during CPR.

The major limitation of the study is the sample size. It is not large enough to evaluate the impact of the study intervention on the neurological prognosis.
However, the primary endpoint was a safety issue and because of absence of previous data about this topic, sample size was not possible to calculate.

In conclusion, the intravenous administration of non-fractionated heparin in a dose of 10000 units during CPR for outof-hospital cardiac arrest in patients with supposed AMI was a safe procedure. We did not observe any improvement in patients' prognosis. Though the procedure proved safe, we recommend postponing the administration of heparin until the return of spontaneous circulation, the assessment of clinical state and the recording of a twelve-lead ECG and until further specification of the diagnosis or of acute coronary syndrome or pulmonary embolism. Our pilot study presents more questions than answers. However, it is important from our point of view that the study proved the safety and simplicity of the procedure.

\section{ACKNOWLEDGMENTS}

The study was supported by the research grants IGA MHCZ NR/7970-3, IGA MHCZ NS10383-2/2009 and by a research project MZO 00179906 .

\section{REFERENCES}

1. Nolan JP, Neumar RW, Adrie C, Aibiki M, Berg RA, Böttiger BW, et al. Post-cardiac arrest syndrome: epidemiology, pathophysiology, treatment, and prognostication. A Scientific Statement from the International Liaison Committee on Resuscitation; the American Heart Association Emergency Cardiovascular Care Committee; the Council on Cardiovascular Surgery and Anesthesia; the Council on Cardiopulmonary, Perioperative, and Critical Care; the Council on Clinical Cardiology; the Council on Stroke. Resuscitation 2008;79:350-79.

2. Binks A, Nolan JP. Post-cardiac arrest syndrome. Minerva Anestesiol 2010;76:362-8.

3. Böttiger BW, Martin E. Thrombolytic therapy during cardiopulmonary resuscitation and the role of coagulation activation after cardiac arrest. Curr Opin Crit Care 2001;7:176-83.

4. Adams JA. Targeting the Endothelium. Signa vitae 2010;5(Suppl 1):17-23.

5. Böttiger BW, Arntz HR, Chamberlain DA, Bluhmki E, Belmans A, Danays T, et al.; TROICA Trial Investigators; European Resuscitation Council Study Group. Thrombolysis during resuscitation for out-of-hospital cardiac arrest. N Engl J Med 2008;359:2651-62.

6. Nolan JP, Deakin CD, Soar J, Böttiger BW, Smith G; European Resuscitation Council. European Resuscitation Council guidelines for resuscitation 2005. Section 4. Adult advanced life support. Resuscitation 2005;67 Suppl 1:S39-86.

7. Deakin CD, Nolan JP, Soar J, Sunde K, Koster RW, Smith GB,et al. European Resuscitation Council Guidelines for Resuscitation 2010 Section 4. Adult advanced life support. Resuscitation 2010;81:1305-52.

8. Stein PD, Hull RD, Raskob G. Risks for major bleeding from thrombolytic therapy in patients with acute pulmonary embolism. Consideration of non-invasive management. Ann Intern Med 1994;121:313-7.

9. Nolan JP, Morley PT, Hoek TL, Hickey RW, Advancement Life support Task Force of the International Liaison committee on Resuscitation. 
Therapeutic hypothermia after cardiac arrest. An advisory statement by the Advancement Life support Task Force of the International Liaison committee on Resuscitation. Resuscitation 2003;57:231-5.

10. Holmberg M, Holmberg S, Herlitz J. The problem of out-of-hospital cardiac-arrest prevalence of sudden death in Europe today. Am J Cardiol 1999;83:88D-90D.

11. Atwood C, Eisenberg MS, Herlitz J, Rea TD. Incidence of EMS-treated out-of-hospital cardiac arrest in Europe. Resuscitation 2005;67:75-80.

12. Böttiger BW, Motsch J, Böhrer H, Böker T, Aulmann M, Nawroth PP, et al. Activation of blood coagulation after cardiac arrest is not balanced adequately by activation of endogenous fibrinolysis. Circulation 1995;92:2572-8.

13. Varriale P, Maldonado JM. Echocardiographic observations during in-hospital cardiopulmonary resuscitation. Crit Care Med 1997;25:1717-20

14. Ruiz-Bailén M, Morante-Valle A, Castillo-Rivera A, Pintor-Mármol A, Ramos-Cuadra JA, Expósito-Ruiz M, et al. Echocardiographic observations during in-hospital cardiopulmonary resuscitation. Resuscitation 2006;71:264-6

15. Memtsoudis SG, Rosenberger P, Loffler M, Eltzschig HK, Mizuguchi A, Shernan

SK, et al. The usefulness of transesophageal echocardiography during intraoperative cardiac arrest in noncardiac surgery. Anesth Analg 2006;102:1653-7.

16. Schneider A, Böttiger BW, Popp E. Cerebral resuscitation after cardiocirculatory arrest. Anesth Analg 2009;108:971-9.

17. Böttiger BW, Böhrer H, Böker T, Motsch J, Aulmann M, Martin E. Platelet factor 4 release in patients undergoing cardiopulmonary resuscitation--can reperfusion be impaired by platelet activation? Acta Anaesthesiol Scand 1996;40:631-5.

18. Gando S, Nanzaki S, Morimoto Y, Kobayashi S, Kemmotsu O. Out-of-hospital cardiac arrest increases soluble vascular endothelial adhesion molecules and neutrophil elastase associated with endothelial injury. Intensive Care Med 2000;26:38-44.

19. Gando S, Kameue T, Nanzaki S, Igarashi M, Nakanishi Y. Platelet activation with massive formation of thromboxane A2 during and after cardiopulmonary resuscitation. Intensive Care Med 1997;23:71-6

20. Moliterno DJ. Advances in antiplatelet therapy for ACS and PCI. J Interv Cardiol 2008;21(Suppl 1):S18-S24.

21. Malý M. Atherothrombosis: the antiaggregant treatment. Cor vasa 2009;51(Suppl 1):19-21.

22. Spiel AO, Frossard M, Mayr FB, Kliegel A, Janata A, Uray T, et al. Pronounced platelet hyperfunction in patients with cardiac arrest achieving restoration of spontaneous circulation. Crit Care Med 2009;37:975-9.

23. Kern KB, Sasaoka T, Higashi H, Hilwig RW, Berg RA, Zuercher M. Post resuscitation myocardial microcirculatory dysfunction is ameliorated with platelet glycoprotein IIb/IIla inhibition. Circulation 2007;116:II630 -II631.

24. Böttiger BW, Bode C, Kern S, Gries A, Gust R, Glätzer R, et al. Efficacy and safety of thrombolytic therapy after initially unsuccessful cardiopulmonary resuscitation: a prospective clinical trial. Lancet 2001;357:1583-5.

25. Fatovich DM, Dobb GJ, Clugston RA. A pilot randomised trial of thrombolysis in cardiac arrest (The TICA trial). Resuscitation 2004;61:309-13

26. Bozeman WP, Kleiner DM, Ferguson KL. Empiric tenecteplase is associated with increased return of spontaneous circulation and short term survival in cardiac arrest patients unresponsive to standard interventions. Resuscitation 2006;69:399-406.

27. Li X, Fu QL, Jing XL, Li YJ, Zhan H, Ma ZF, et al. A meta-analysis of cardiopulmonary resuscitation with and without the administration of thrombolytic agents. Resuscitation 2006;70:31-6.

28. Snyder-Ramos SA, Motsch J, Martin E, Böttiger BW. Thrombolytic therapy during cardiopulmonary resuscitation. Minerva Anestesiol 2002;68:186-91.

29. Spöhr F, Böttiger BW. Thrombolytic therapy during or after cardiopulmonary resuscitation. Efficacy and safety of a new therapeutic approach. Minerva Anestesiol 2003;69: 357-64.

30. Spöhr F, Böttiger BW. Thrombolytics in CPR. Current advantages in cardiopulmonary resuscitation. Minerva Anestesiol 2005;71:291-6.

31. Spöhr F, Böttiger BW. Safety of thrombolysis during cardiopulmonary resuscitation. Drug Saf 2003;26:367-79.

32. Crowell JW, Sharpe GP, Lambright RL, Read WL. The mechanism of death after resuscitation following acute circulatory failure. Surgery 1955;38:696-702.

33. Gaszyński W. The use of protease inhibitor (trasylol) and heparin in cardiorespiratory resuscitation. I. Studies of the blood clotting system. Anaesth Resusc Intensive Ther 1975;3:125-34.

34. Matyal R, Grinberg AO, Panzica PJ, Darke M, Lerner AB, Fisher L, et al. Heparin administration during cardiopulmonary resuscitation. J Cardiothorac Vasc Anesth 2008;22:861-3.

35. Spaulding CM, Joly LM, Rosenberg A, Monchi M, Weber SN, Dhainaut JF, et al. Immediate coronary angiography in survivors of out-ofhospital cardiac arrest. N Engl J Med 1997;336:1629-33

36. Kuisma M, Alaspää A. Out-of-hospital cardiac arrests of non-cardiac origin. Epidemiology and outcome. Eur Heart J 1997;18:1122-8. 